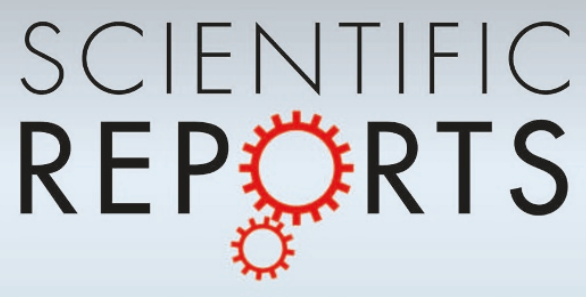

OPEN

SUBJECT AREAS:

ELECTRONIC PROPERTIES

AND MATERIALS

TWO-DIMENSIONAL MATERIALS

Received

11 October 2013

Accepted

3 January 2014

Published

22 January 2014

Correspondence and requests for materials should be addressed to Z.-M.L. (liaozm@pku. edu.cn)

* These authors contributed equally to this work.

\section{High-Mobility $\mathrm{Bi}_{2} \mathrm{Se}_{3}$ Nanoplates} Manifesting Quantum Oscillations of Surface States in the Sidewalls

\author{
Yuan Yan ${ }^{*}$, Li-Xian Wang ${ }^{1 *}$, Xiaoxing Ke ${ }^{2 *}$, Gustaaf Van Tendeloo ${ }^{2}$, Xiao-Song Wu ${ }^{1,3}$, Da-Peng Yu $u^{1,3}$ \\ \& Zhi-Min Liao ${ }^{1,3}$ \\ ${ }^{1}$ State Key Laboratory for Mesoscopic Physics, Department of Physics, Peking University, Beijing 100871, P.R. China, ${ }^{2}$ EMAT \\ (Electron Microscopy for Materials Science), University of Antwerp, Groenenborgerlaan 171, 2020 Antwerp, Belgium, \\ ${ }^{3}$ Collaborative Innovation Center of Quantum Matter, Beijing, P.R. China.
}

Magnetotransport measurements of topological insulators are very important to reveal the exotic topological surface states for spintronic applications. However, the novel properties related to the surface Dirac fermions are usually accompanied by a large linear magnetoresistance under perpendicular magnetic field, which makes the identification of the surface states obscure. Here, we report prominent Shubnikov-de Haas $(\mathrm{SdH})$ oscillations under an in-plane magnetic field, which are identified to originate from the surface states in the sidewalls of topological insulator $\mathrm{Bi}_{2} \mathrm{Se}_{3}$ nanoplates. Importantly, the $\mathrm{SdH}$ oscillations appear with a dramatically weakened magnetoresistance background, offering an easy path to probe the surface states directly when the coexistence of surface states and bulk conduction is inevitable. Moreover, under a perpendicular magnetic field, the oscillations in Hall conductivity have peak-to-valley amplitudes of $2 \mathrm{e}^{2} / \mathrm{h}$, giving confidence to achieve a quantum Hall effect in this system. A cross-section view of the nanoplate shows that the sidewall is (015) facet dominant and therefore forms a $58^{\circ}$ angle with regard to the top/ bottom surface instead of being perpendicular; this gives credit to the surface states' behavior as two-dimensional transport.

. hree dimensional (3D) topological insulators have been demonstrated to possess unique physical properties ${ }^{1-4}$. Among the family of $3 \mathrm{D}$ topological insulators, $\mathrm{Bi}_{2} \mathrm{Se}_{3}$ is regarded as an ideal platform to explore the exotic properties of Dirac fermions on the surface ${ }^{5-7}$. Using angle resolved photoemission spectroscopy (ARPES), the spin-momentum locked surface states are firmly confirmed ${ }^{8-10}$. To further shed light on future applications on spintronic devices, it is crucial to investigate the peculiar surface states via transport measurements. However, even with a large bulk bandgap, the conductance contributed from bulk is notable in as-grown $\mathrm{Bi}_{2} \mathrm{Se}_{3}$ samples, which makes identification of the surface states by transport measurements very challenging ${ }^{11}$. This prominent bulk contribution is related to the excess carriers in bulk due to Se vacancies in $\mathrm{Bi}_{2} \mathrm{Se}_{3}$. To reduce the excess carriers, chemical doping ${ }^{12,13}$ and carrier exhaustion by applying a gate voltage ${ }^{14-16}$ are proved to be efficient methods. However, low bulk carrier concentration is still insufficient to make surface states overwhelm the bulk in magnetotransport measurements, because the expected signals from the surface states are always accompanied by a large positive magnetoresistance (MR) background ${ }^{17}$. Quantum oscillations are clearly observed in topological insulator nanostructures, such as Shubnikov-de Haas ( $\mathrm{SdH}$ ) oscillations ${ }^{18-20}$, Aharonov-Bohm oscillations ${ }^{21}$ and universal conductance fluctuations ${ }^{22}$, which can help to discern the nontrivial surface state transport from the inevitable bulk contribution. Through analysis of the SdH oscillation, the additional $\pi$ Berry phase can be detected, which is a distinguished signature of two dimensional Dirac fermions ${ }^{23}$. Nevertheless, it is important and urgent to diminish the bulk MR background in detecting surface states via $\mathrm{SdH}$ oscillations $s^{24,25}$. On the other hand, it is also interesting to know if the surface states in the narrow sidewalls of $\mathrm{Bi}_{2} \mathrm{Se}_{3}$ nanoplate can present exotic quantum transport properties.

In this work, we report significant $\mathrm{SdH}$ oscillations from the sidewalls of $\mathrm{Bi}_{2} \mathrm{Se}_{3}$ nanoplates with the bulk background of a two-dimensional (2D) positive MR substantially weakened. Additionally, the $\pi$ Berry phase is extracted from the analysis of the SdH oscillations, which is ascribed to the 2D Dirac fermions from the surface states in the sidewalls of the $\mathrm{Bi}_{2} \mathrm{Se}_{3}$ nanoplates. Meanwhile, by changing the orientation of the magnetic field referring to the substrate plane, the coexistence of two $\mathrm{SdH}$ oscillation modes from sidewall and top/bottom surfaces, respectively, is gradually revealed. 

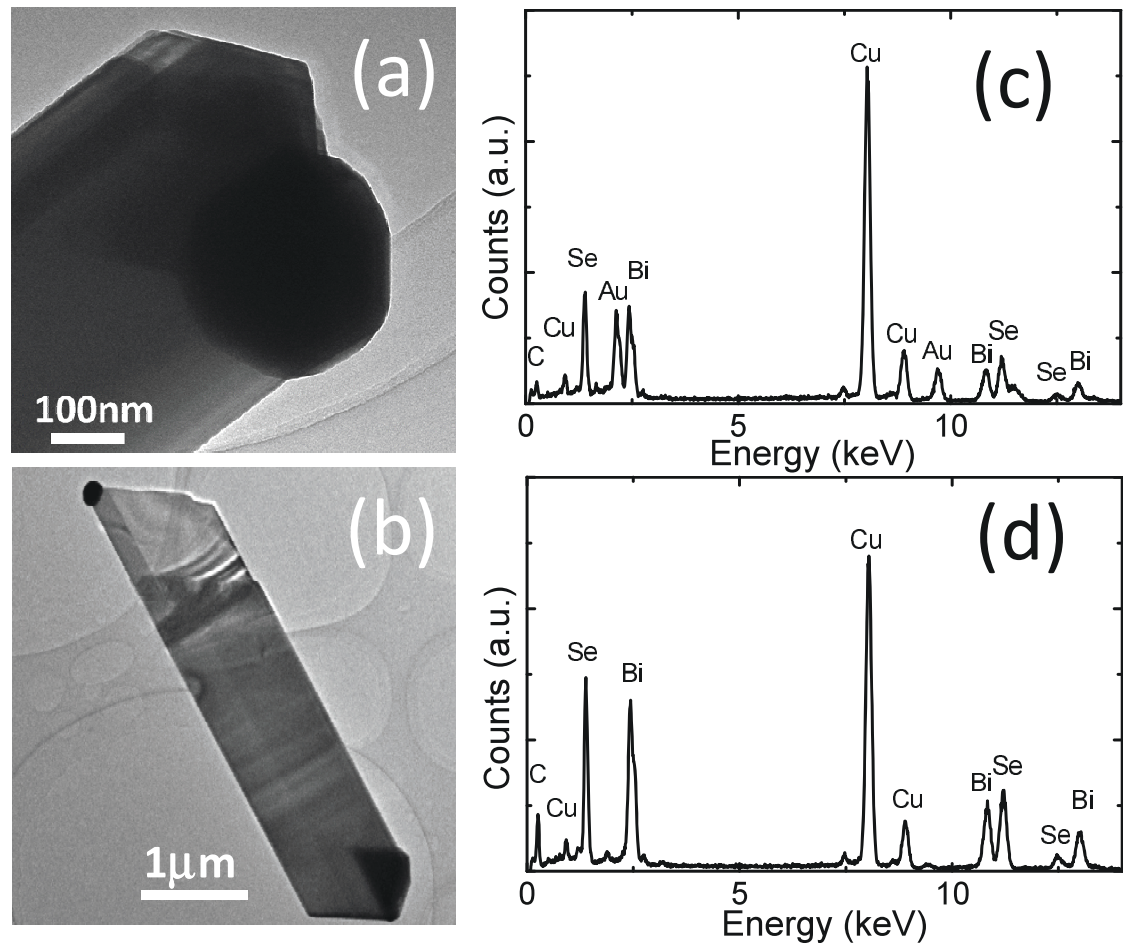

Figure $1 \mid$ TEM and EDS characterizations. (a, b) TEM images of a typical $\mathrm{Bi}_{2} \mathrm{Se}_{3}$ nanoribbon and nanoplate with gold particle at the tip-edge, respectively. (c, d) EDS spectra obtained at the regions with the gold particle and without the gold particle, respectively.

\section{Results}

The $\mathrm{Bi}_{2} \mathrm{Se}_{3}$ nanostructures were grown by the vapor-liquid-solid mechanism with gold as catalyst. Gold particles can be found at the tip-edge of the $\mathrm{Bi}_{2} \mathrm{Se}_{3}$ nanoribbons and nanoplates, as shown by the transmission electron microscopy (TEM) images in Figures 1(a) and 1(b). Corresponding energy-dispersive X-ray spectroscopy (EDS) spectra collected from the tip region and body region are shown in Figures 1(c) and 1(d), respectively, which reveal that the gold particles are only present at the tip region. Figure 2(a) shows a typical $\mathrm{Bi}_{2} \mathrm{Se}_{3}$ nanoplate device and a schematic diagram of the orientation of the applied magnetic field. Since the electrical contacts are made on the central part of the $\mathrm{Bi}_{2} \mathrm{Se}_{3}$ nanoplate, the gold catalyst particle at the tip does not influence the transport properties. The $c$ axis of the $\mathrm{Bi}_{2} \mathrm{Se}_{3}$ nanoplate is perpendicular to the substrate plane. $\theta$ was defined as the angle between the orientation of the magnetic field and the $c$ axis of the $\mathrm{Bi}_{2} \mathrm{Se}_{3}$ nanoplate. $\varphi$ was defined as the angle between the orientation of the in-plane magnetic field $\left(\theta=90^{\circ}\right)$ and the direction perpendicular to the long-side edge of the $\mathrm{Bi}_{2} \mathrm{Se}_{3}$ nanoplate. The thickness, length between the two longitudinal voltage leads, and the width of the two Hall voltage leads of the $\mathrm{Bi}_{2} \mathrm{Se}_{3}$ nanoplate device are $140 \mathrm{~nm}, 1.48 \mu \mathrm{m}$ and $2.26 \mu \mathrm{m}$, respectively. Applying a magnetic field perpendicular to the substrate plane, Hall measurements were performed (Figure 2(b)). From the Hall measurements, the carrier concentration and carrier mobility were calculated and presented in Figs. 3(a) and 3(b), respectively. It can be seen that the carrier concentration decreases and the carrier mobility increases with decreasing temperature down to $30 \mathrm{~K}$, and only change a little bit from $30 \mathrm{~K}$ to $2 \mathrm{~K}$, showing a semiconductor-like behavior. At $2 \mathrm{~K}$, a carrier concentration $n_{\text {Hall }}=5.2 \times 10^{18} \mathrm{~cm}^{-3}$ and a carrier mobility $\mu_{\text {Hall }}=8800 \mathrm{~cm}^{2} /$ Vs are obtained.

When rotating the magnetic field into the substrate plane $(\mathrm{B} \perp \mathbf{c}, \theta$ $=90^{\circ}$ ), the orientation of the in-plane magnetic field forms an angle $\varphi=50^{\circ}$ with the direction perpendicular to the side edge of the $\mathrm{Bi}_{2} \mathrm{Se}_{3}$ nanoplate. In order to simplify the analysis, we only consider the component of the in-plane magnetic field that perpendicular to the long-side edge of the $\mathrm{Bi}_{2} \mathrm{Se}_{3}$ nanoplate as the effective magnetic
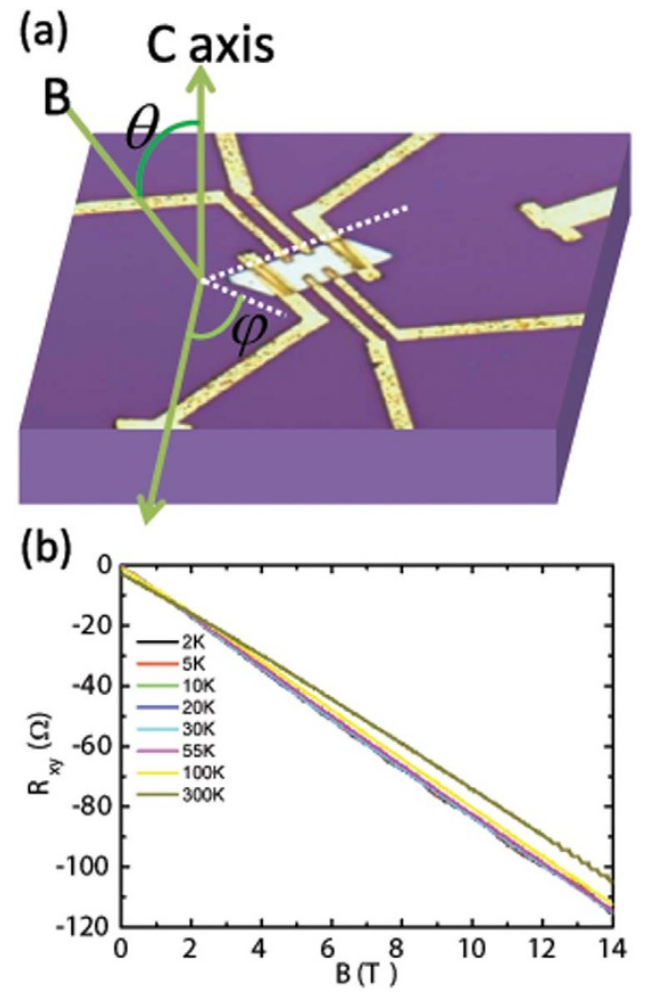

Figure $2 \mid$ Device structure and Hall measurements. (a) Optical image of a typical $\mathrm{Bi}_{2} \mathrm{Se}_{3}$ nanoplate device and a schematic of the orientation of the applied magnetic field. The length between the two inner longitudinal voltage leads, and the width of the two Hall voltage leads of the $\mathrm{Bi}_{2} \mathrm{Se}_{3}$ nanoplate device are $1.48 \mu \mathrm{m}$ and $2.26 \mu \mathrm{m}$, respectively. (b) The Hall resistance of the $\mathrm{Bi}_{2} \mathrm{Se}_{3}$ nanoplates at different temperatures under a perpendicular magnetic field. 

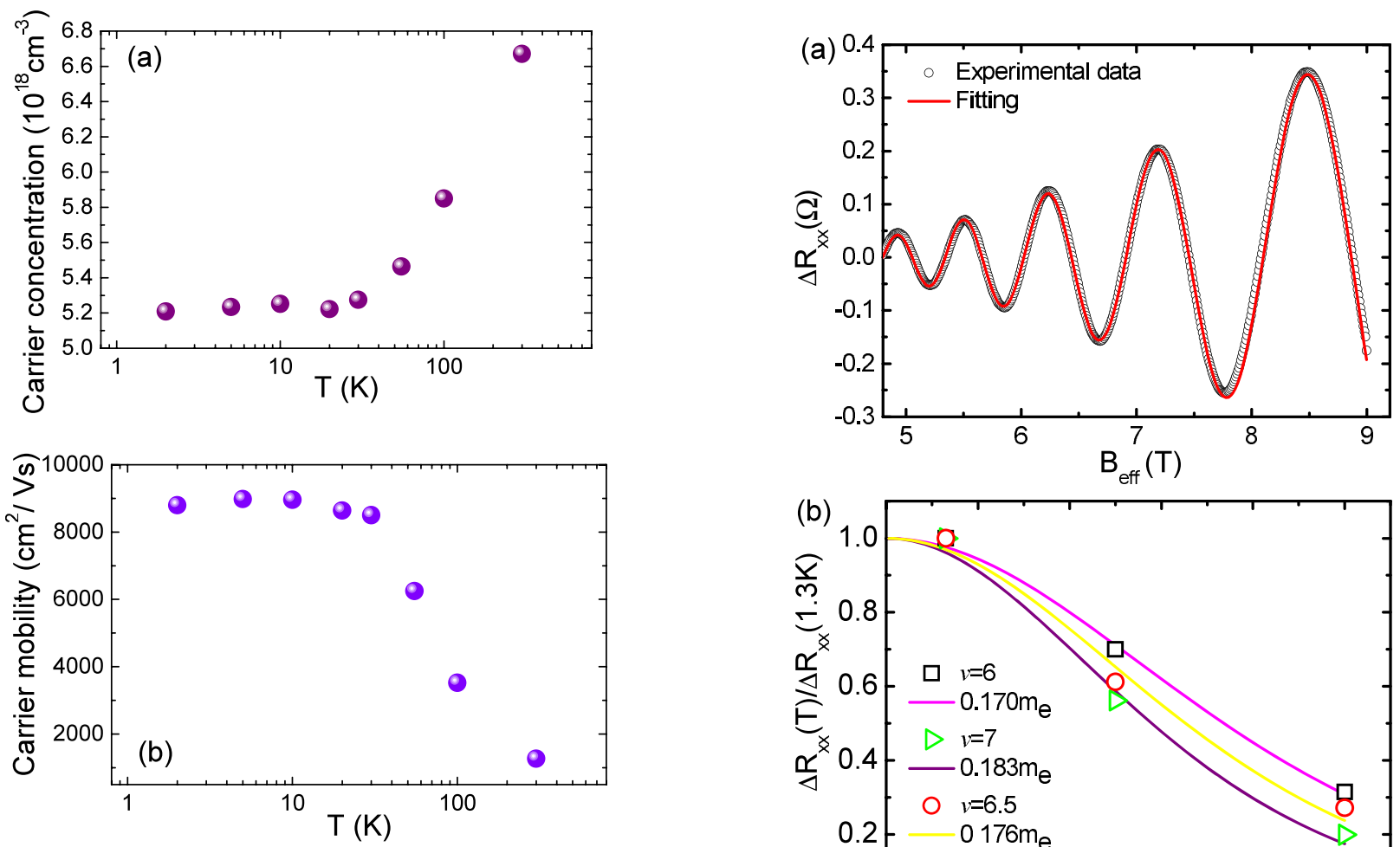

Figure $3 \mid$ Temperature dependent carrier density and mobility. (a) Carrier concentration and (b) mobility from the Hall measurements under different temperatures.
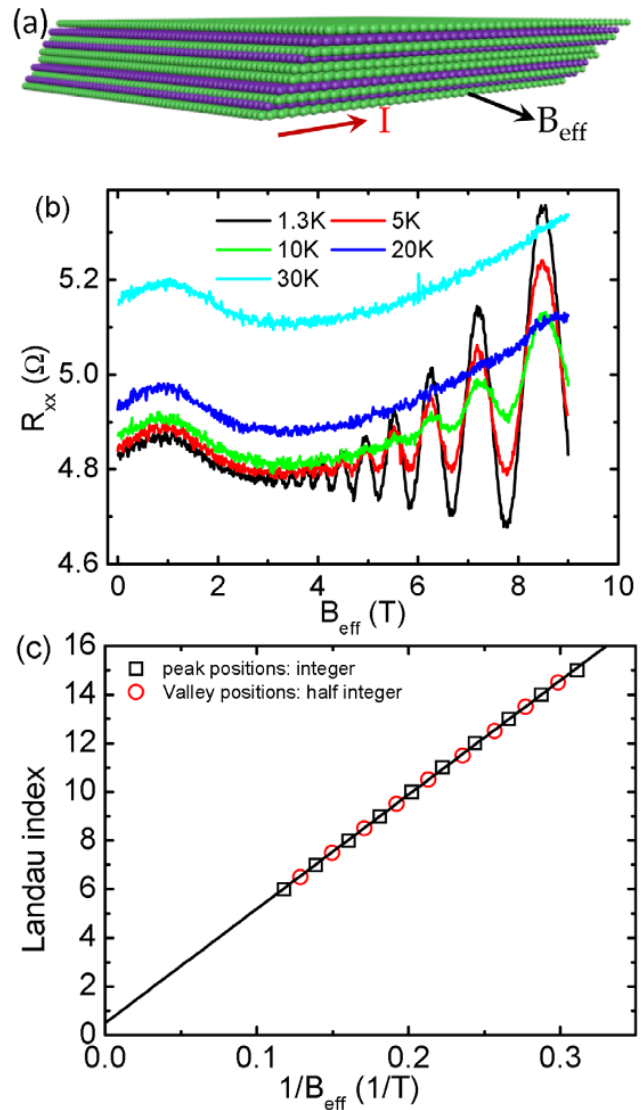

Figure $4 \mid$ Magnetotransport under in-plane magnetic field. (a)

Schematic of the in-plane magnetic field referring to the sidewall (Se: green spheres; Bi: purple spheres). (b) Plot of longitudinal resistance $R_{x x}$ versus $B_{\text {eff }}$ under in-plane magnetic field at different temperatures. (c) Landaulevel fan diagram for the Shubnikov-de Haas oscillation in $\mathrm{R}_{\mathrm{xx}}$ at the lowest temperature $1.3 \mathrm{~K}$. 

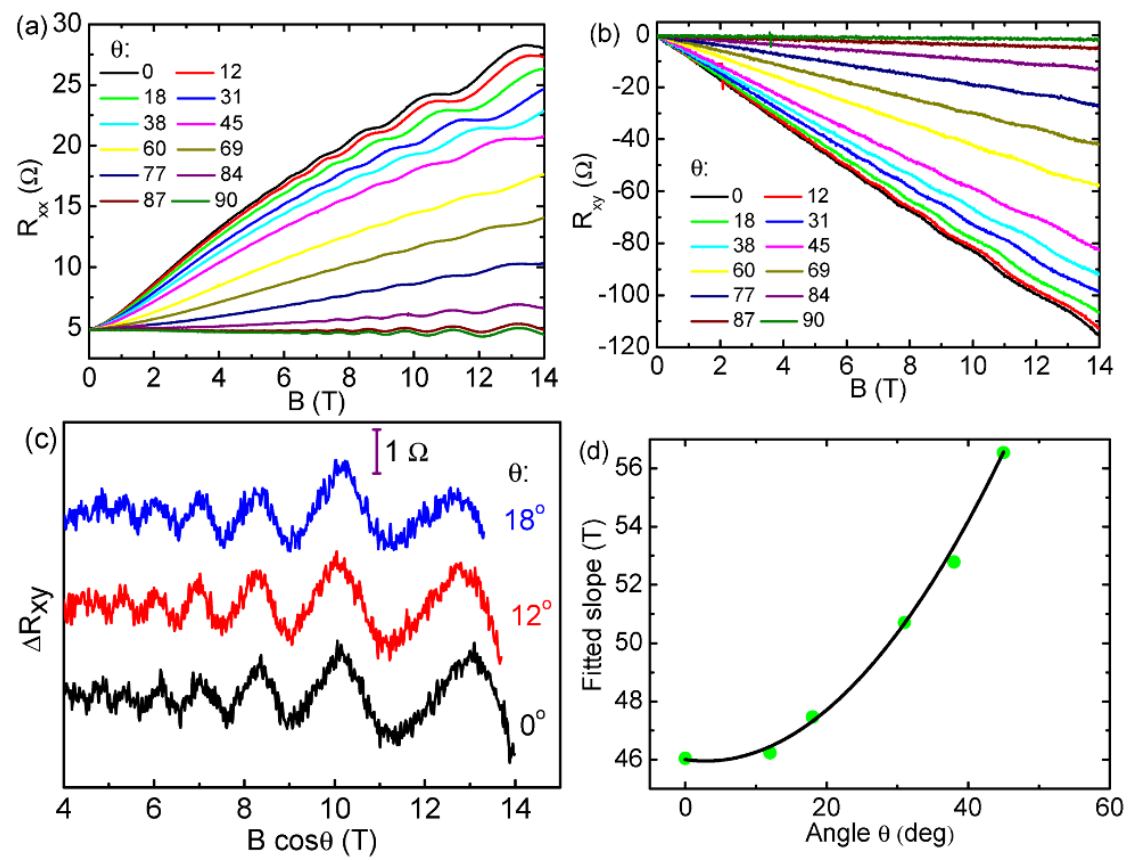

Figure $6 \mid$ Angle $\boldsymbol{\theta}$ dependent magnetotransport. (a) Longitudinal resistance $R_{x x}$ and (b) Hall resistance $R_{x y}$ as a function of the applied magnetic field $B$ under different orientations described by the angle $\theta$, at a temperature of $2 \mathrm{~K}$. (c) Oscillations of the Hall resistance at $2 \mathrm{~K}$ and at different angle $\theta$. (d) Fitted slope of the Landau level fan diagram derived from $R_{x y}$ as a function of the angle $\theta$; the solid line gives a good fitting with $1 / \cos \theta$.

It is well known that the Onsager semiclassical quantization relation can be expressed in the form $A_{N}=\frac{2 \pi e}{\hbar} B\left(N_{\min }+\gamma-\frac{1}{2}\right)$, where $\gamma$ is directly related to Berry phase $\beta$ by $\gamma-\frac{1}{2}=\frac{-\beta}{2 \pi}, N_{\text {min }}$ indexes the $N_{t h}$ minimum in conductance $G_{x x}$ (Fermi level locates between Landau levels), and $A_{N}$ stands for the orbital area of electrons in $k$ space $^{26}$. Here, in the absence of a transversal resistance $R_{x y}$ under an in-plane magnetic field, only the longitudinal resistance of $R_{x x}$ is available for the $\mathrm{SdH}$ oscillation analysis. Given that the area of sidewall is very small compared to top/bottom surface area (the ratio between width and height of our device is larger than $15: 1$ ), the conductance in the sidewall channel is much smaller than the conductance from the top/bottom surfaces or bulk channels. In this situation, the $N_{t h}$ maximum in $R_{x x}$ matches well with the $N_{t h}$ minimum in $G_{x x}$ for $R_{x x}=G_{x x} /\left[G_{x x}{ }^{2}+G_{x y}{ }^{2}\right] \sim 1 / G_{x x}$ proposed by $\mathrm{J}$. Xiong et al. ${ }^{27}$. Therefore, it is reasonable to assign the peak positions in $R_{x x}$ to the integer Landau index when analyzing the $\mathrm{SdH}$ oscillations here. By using the Landau-level (LL) diagram fan shown in Fig. 4(c), the extrapolated Landau index $N$ at extreme field limit $(1 / \mathrm{B} \rightarrow 0)$ is obtained to be $0.5($ i.e. $\gamma=0)$ exactly, which implies

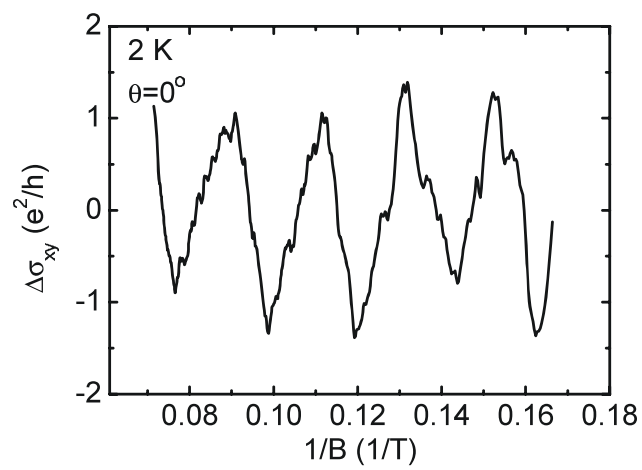

Figure $7 \mid$ Oscillations in $\sigma_{x y}$ as a function of $1 / B$ under perpendicular magnetic field. the additional Berry phase $\beta=-2 \pi \times\left(\gamma-\frac{1}{2}\right)=\pi$, as a direct evidence of 2D Dirac fermions. To provide dual evidences for Dirac electrons, we perform further analysis on the $\mathrm{SdH}$ oscillations of $\Delta R_{x x}$. The magnetic field dependence of $\mathrm{SdH}$ oscillation in $\mathrm{R}_{\mathrm{xx}}$ can be described as

$$
\left.\Delta R(B)=A \exp (-\pi / \mu \mathrm{B}) \cos \left[2 \pi\left(\mathrm{B}_{F} / \mathrm{B}\right)+\beta\right]\right],
$$

where $\mu$ is the quantum mobility and $B_{F}$ is the oscillation frequency ${ }^{26}$. Figure 5(a) shows that the $\Delta R_{x x}$ fits eq. (1) quite well, yielding $\mu=$ $1300 \mathrm{~cm}^{2} / V s, B_{F}=47 T$, and $\beta=0.9 \pi$. The phase $\beta$ is quite close to the expected $\pi$ Berry phase for Dirac electrons, in agreement with previous results from the LL fan diagram. The fitted $B_{F}$ is also consistent with the obtained slope $B_{F}^{\text {in-plane }}(46.9 \mathrm{~T})$ in the LL fan diagram, which implies $n_{S d H}^{\text {in-plane }}=\frac{e}{h} B_{F}^{\text {in-plane }}=1.1 \times 10^{12} \mathrm{~cm}^{-2}$ (per spin) and a corresponding Fermi wave-vector $k_{F}^{\text {in-plane }}=$ $\left(4 \pi n_{S d H}\right)^{1 / 2}=3.8 \times 10^{6} \mathrm{~cm}^{-1}$.

From the temperature dependent amplitudes of the $\mathrm{SdH}$ oscillations under an in-plane magnetic field, the cyclotron mass $m_{c y c}$ can be obtained by fitting $\Delta R_{x x}(T) / \Delta R_{x x}(1.3 \mathrm{~K})$ versus temperature $T$ using the function of $\lambda(T) / \sinh \lambda(T)$, where $\lambda(T)=\frac{2 \pi^{2} k_{B} T}{\hbar e B} m_{c y c}$ and $k_{B}$ is the Boltzmann constant ${ }^{27}$. The $m_{c y c} \sim 0.17 \mathrm{~m}_{\mathrm{e}}$ is extracted from the fitting in Fig. 5(b), consistent with previous reports ${ }^{1928,29}$. According to the Dingle plot in Fig. 5(c), the lifetime of electrons between two successive scattering events is calculated to be $\tau^{\text {in-plane }}=$ $1.38 \times 10^{-13} s$ at $1.3 \mathrm{~K}$, and the corresponding Dingle temperature $T_{D}^{\text {in-plane }}=8.81 \mathrm{~K}$.

As the magnetic field is perpendicular to the top/bottom surfaces of the nanoplate $\left(\theta=0^{\circ}\right)$, a large positive $\mathrm{MR}(\sim 500 \%)$ is observed, as shown in Fig. 6(a). SdH oscillations clearly appear on the large positive MR background. The values $B_{F}^{\text {perpendicular }}=47.3 T$ and $n_{S d H}^{\text {perpendicular }}=1.1 \times 10^{12} \mathrm{~cm}^{-2}$ were achieved. As rotating the magnetic field to deflect from the perpendicular direction, i.e., from $\theta$ $=0^{\circ}$ to $\theta=60^{\circ}$, at the same $\mathrm{B}$, the oscillation amplitude and the positive $\mathrm{MR}$ decreases, indicating a $2 \mathrm{D}$ transport behavior under 

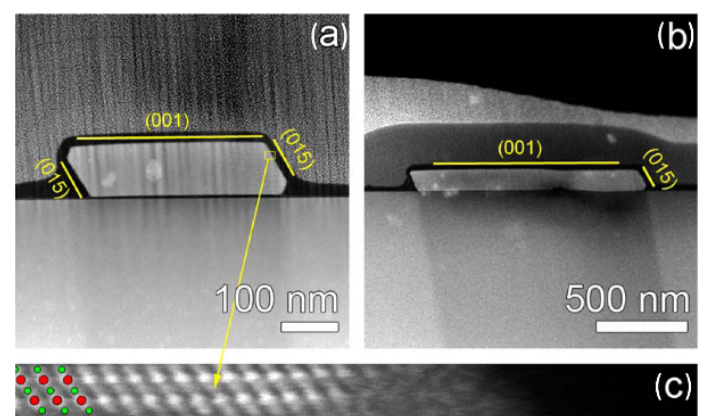
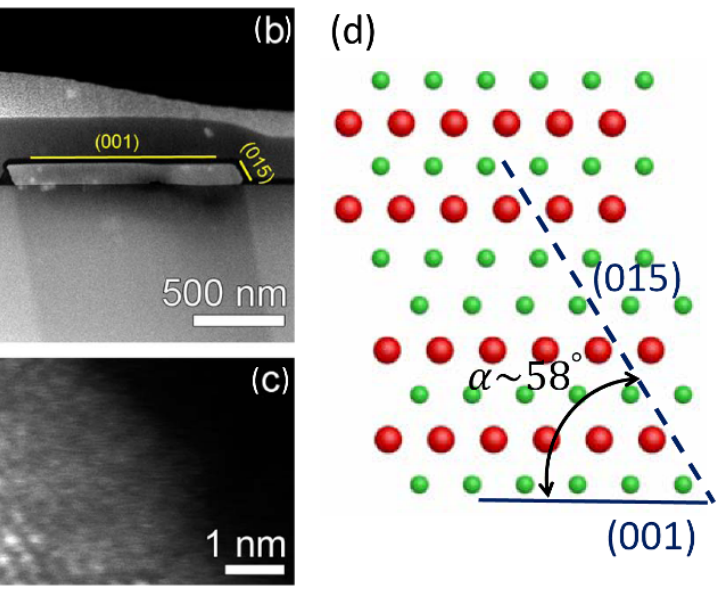

Figure 8 HAADF-STEM images of $\mathrm{Be}_{2} \mathrm{Se}_{3}$ nanoplates in cross-section. (a) and (b) show two individual nanoplates cross-section geometry at low magnification. Although they have different size, the geometries are mostly similar. Upper/bottom surfaces are (001) facet, whereas sidewalls have (015) facets, with an angle of $\sim 58$ degrees with regard to the upper/bottom surface. (c) High resolution HAADF-STEM image of the sidewall surface at atomic resolution, viewed along the [100] zone axis. Bi columns are indicated by red dots and Se columns are indicated by green dots. (d) Schematic drawing of $\mathrm{Bi}_{2} \mathrm{Se}_{3}$ atomic arrays viewed along the [100] zone axis (Bi atoms: red spheres; Se atoms: green spheres).

perpendicular field. At an angle $\theta$, the magnetic field has both vertical and in-plane components, and the surface states from both the top/ bottom surfaces and the sidewalls can contribute to the $\mathrm{SdH}$ oscillations. To avoid this complexity, we measured the transversal Hall resistance $R_{x y}$ at $2 \mathrm{~K}$, as presented in Fig. 6(b), which should not be affected by the sidewall conductance. Clear oscillations were observed in the $R_{x y}$ (Fig. 6(c)). The $B_{F}$ from the $R_{x y}$ LL fan diagram slope as a function of $\theta$ is well fitted by $1 / \cos \theta$, as shown in Fig. 6(d), which confirms the $2 \mathrm{D}$ properties of the $\mathrm{SdH}$ oscillations under perpendicular field. Therefore, the 3D bulk transport does not contribute to the observed $\mathrm{SdH}$ oscillations, and the $\mathrm{SdH}$ oscillations under in-plane magnetic field should originate from 2D surface transport in the sidewalls. Moreover, under a perpendicular magnetic field, the oscillations in Hall conductivity $\sigma_{x y}$ display a peak-tovalley amplitude of $2 \mathrm{e}^{2} / \mathrm{h}$, as shown in Fig. 7. The amplitudes of $\Delta \sigma_{x y}$ do not decay with varying magnetic field, indicating evidence for a quantized Hall conductivity.

It is worth noting that, as rotating the magnetic field deflected from in-plane direction, i.e. from $\theta=90^{\circ}$ to $\theta=69^{\circ}$, the oscillation peaks move towards the low magnetic field part in Figure 6(a). To further reveal the $\mathrm{SdH}$ oscillations from the surface states of the sidewalls, we have examined the sample geometry. We have made cross sections of two other similar $\mathrm{Bi}_{2} \mathrm{Se}_{3}$ nanoplates, as shown in Figure 8. It is found that the sidewall surface is a bevel, which is believed to be responsible for the observation that the quantum oscillation peaks move toward the low magnetic field part as $\theta$ deviates from $90^{\circ}$ to $69^{\circ}$. High angle annular dark field scanning transmission electron microscopy (HAADF-STEM) at atomic resolution has revealed that the side-wall is (015) facet dominant and therefore forms an angle of approximately $\alpha \sim 58^{\circ}$ relative to the top/bottom surface as shown in Fig. $8 \mathrm{c}-\mathrm{d}$. According to the sample geometry as revealed by TEM cross-section, we performed a detailed analysis on the $\mathrm{SdH}$ oscillations as the magnetic field is near the substrate plane. As shown in Figure 9, the index field $B_{n}$ scales as $1 / \cos (\theta-\alpha)$ by considering the $2 \mathrm{D}$ transport of the surface states in the sidewalls along the bevel, and $\alpha=56^{\circ}$ is obtained for this device, which matches well with the (015) dominant facets as revealed by the HAADF-STEM results.

\section{Discussion}

It should be noticed that $n_{S d H}^{\text {perpendicular }}$ is higher than the carrier concentration from the Hall measurement $5.2 \times 10^{11} \mathrm{~cm}^{-2}\left(n_{\text {Hall }}{ }^{3 D}\right.$ per quintuple layer), suggesting that the $\mathrm{SdH}$ oscillations do not originate from the 2D bulk transport. The high surface carrier concentrations may be due to the n-type doping from the surface oxide layer ${ }^{30}$, which can be noticed on the HAADF-STEM image of the cross-section sample in Fig. 8c.

In conclusion, we have studied the quantum transport of individual $\mathrm{Bi}_{2} \mathrm{Se}_{3}$ nanoplates, which are of high carrier mobility $\sim 8800 \mathrm{~cm}^{2} / \mathrm{Vs}$ at low temperatures. Under a perpendicular magnetic field, $\mathrm{SdH}$ oscillations accompanied by a large positive linear magnetoresistance up to $500 \%$, and a quantized Hall conductivity with a value of $\sim 1 e^{2} / h$ per surface were observed. Both the $\mathrm{SdH}$ oscillations and the large positive MR show two-dimensional transport properties via angle dependent magnetotransport measurements. Most interestingly, $\mathrm{SdH}$ oscillations from the nontrivial surface states residing in the sidewalls of the $\mathrm{Bi}_{2} \mathrm{Se}_{3}$ nanoplates were observed under an in-plane magnetic field. Unlike usually observed $\mathrm{SdH}$ oscillations on the top/bottom surfaces, the oscillations observed from the sidewalls are more prominent for bearing a much weaker MR background, implying a potential, easy path to study the surface states through transport measurements. Moreover, the crosssection view of the sample shows the sidewall is a bevel, which should be considered by analyzing the angular dependence of the magnetotransport.

\section{Methods}

Growth of $\mathrm{Bi}_{2} \mathrm{Se}_{3}$ nanoplates. The $\mathrm{Bi}_{2} \mathrm{Se}_{3}$ nanoplates were synthesized by chemical vapor deposition method in evacuated tube furnace. $\mathrm{Bi}_{2} \mathrm{Se}_{3}$ powders with $99.999 \%$ purity placed in a ceramic boat were located at the center of the tube furnace. The system was first pumped and flashed by Ar gas. The system was then heated to $700^{\circ} \mathrm{C}$ within 30 minutes. Si substrates with a layer of $10 \mathrm{~nm}$-thick gold film were used to collect the products at the downstream of the evaporation source following with $\mathrm{Ar}$ carrier gas.

Device fabrication. The as-grown $\mathrm{Bi}_{2} \mathrm{Se}_{3}$ nanoplates were transferred from the $\mathrm{Si}$ substrate onto an insulating substrate with prefabricated marks. The positions of the desired $\mathrm{Bi}_{2} \mathrm{Se}_{3}$ nanoplates were first recorded by the electron beam lithography (EBL) system. After coating with electron beam resist, EBL was used to pattern the Hall bar geometries. Electrodes of $\mathrm{Cr} / \mathrm{Au}(10 \mathrm{~nm} / 130 \mathrm{~nm})$ were then deposited by electron beam evaporation. After lift-off process, the devices were finalized.

Magnetotransport measurements. The magnetotransport measurements were performed in an Oxford cryostat with variable temperature insert and superconductive magnet. The electrical properties were measured using lowfrequency lock-in techniques with constant current of $1 \mu \mathrm{A}$.

Cross-section samples preparation and STEM characterization. The as-grown $\mathrm{Bi}_{2} \mathrm{Se}_{3}$ nanoplates were first transferred onto a $\mathrm{SrTiO}_{3}$ substrate. After depositing a protective layer of amorphous carbon and subsequently Pt-carbon on the $\mathrm{Bi}_{2} \mathrm{Se}_{3}$ surface, a thin cross-sectional lamella was prepared by Focused Ion Beam (FIB) using 
(a) $\mathrm{N}$
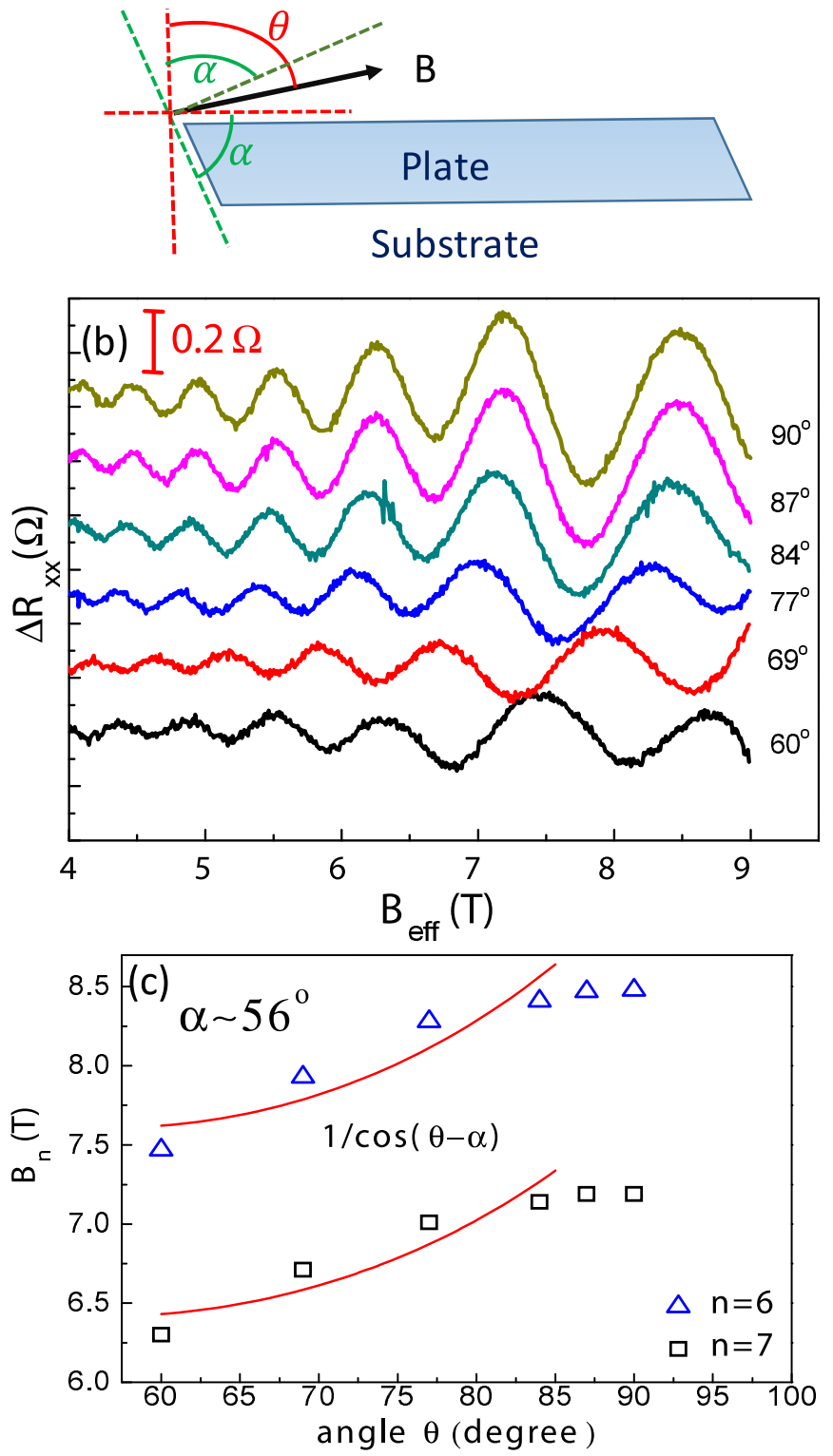

Figure 9 Angular dependence of the $\mathrm{SdH}$ oscillations from sidewall surface states at large angle $\boldsymbol{\theta}$. (a) Scheme of the cross-section view of the $\mathrm{Bi}_{2} \mathrm{Se}_{3}$ nanoplate for illustrating the angle $\alpha$ between the top surface and the side-wall and the angle $\theta$ between the direction of the magnetic field $B$ and the direction perpendicular to the substrate plane. (b) The evolution of the $\mathrm{SdH}$ oscillations as the magnetic field deflects from in-plane direction, i.e. from $\theta=90^{\circ}$ to $\theta=60^{\circ}$. (c) Plot of the peak positions at $\mathrm{n}=6$ and 7 versus the tilted angle $\theta$, a fitting to $1 / \cos (\theta-\alpha)$ obtaining $\alpha=56^{\circ}$, in agreement with the HAADF-STEM results.

a FEI-Helios NanoLab DualBeam system. High-resolution HAADF-STEM characterizations were performed on a FEI Titan microscope fitted with an aberration corrector for the probe-forming lens, operated at $200 \mathrm{kV}$. The STEM convergence semi-angle used was $\sim 21.4 \mathrm{mrad}$.

1. Fu, L., Kane, C. L. \& Mele, E. J. Topological Insulators in Three Dimensions. Phys. Rev. Lett. 98, 106803 (2007).

2. Fu, L. \& Kane, C. L. Topological Insulators with Inversion Symmetry. Phys. Rev. B 76, 045302 (2007).

3. Hsieh, D. et al. Observation of Unconventional Quantum Spin Textures in Topological Insulators. Science 323, 919-922 (2009).

4. Chen, Y. L. et al. Experimental Realization of a Three-Dimensional Topological Insulator, $\mathrm{Bi}_{2} \mathrm{Te}_{3}$. Science 325, 178-181 (2009).
5. Zhang, H. J. et al. Topological Insulators in $\mathrm{Bi}_{2} \mathrm{Se}_{3}, \mathrm{Bi}_{2} \mathrm{Te}_{3}$ and $\mathrm{Sb}_{2} \mathrm{Te}_{3}$ with a Single Dirac Cone on the Surface. Nat. Phys. 5, 438-442 (2009).

6. Xia, Y. et al. Observation of a Large-Gap Topological Insulator Class with a Single Dirac Cone on the Surface. Nat. Phys. 5, 398-402 (2009).

7. Hasan, M. Z. \& Kane, C. L. Topological Insulators. Rev. Mod. Phys. 82, 3045-3067 (2010).

8. Kuroda, K. et al. Hexagonally Deformed Fermi Surface of the 3D Topological Insulator $\mathrm{Bi}_{2} \mathrm{Se}_{3}$. Phys. Rev. Lett. 105, 076802 (2010).

9. Pan, Z.-H. et al. Electronic Structure of the Topological Insulator $\mathrm{Bi}_{2} \mathrm{Se}_{3}$ Using Angle-Resolved Photoemission Spectroscopy: Evidence for a Nearly Full Surface Spin Polarization. Phys. Rev. Lett. 106, 187002 (2011).

10. Hsieh, D. et al. A Tunable Topological Insulator in the Spin Helical Dirac Transport Regime. Nature 460, 1101-1105 (2009).

11. Checkelsky, J. G. et al. Quantum Interference in Macroscopic Crystals of Nonmetallic $\mathrm{Bi}_{2} \mathrm{Se}_{3}$. Phys. Rev. Lett. 103, 246601 (2009).

12. Taskin, A. A., Ren, Z., Sasaki, S., Segawa, K. \& Ando, Y. Observation of Dirac Holes and Electrons in a Topological Insulator. Phys. Rev. Lett. 107, 016801 (2011).

13. Kong, D., Koski, K. J., Cha, J. J., Hong, S. S. \& Cui, Y. Ambipolar Field Effect in SbDoped $\mathrm{Bi}_{2} \mathrm{Se}_{3}$ Nanoplates by Solvothermal Synthesis. Nano Lett. 13, 632-636 (2013).

14. Checkelsky, J. G., Hor, Y. S., Cava, R. J. \& Ong, N. P. Bulk Band Gap and Surface State Conduction Observed in Voltage-Tuned Crystals of the Topological Insulator $\mathrm{Bi}_{2} \mathrm{Se}_{3}$. Phys. Rev. Lett. 106, 196801 (2011).

15. Wang, Z., Qiu, R. L. J., Lee, C. H., Zhang, Z. \& Gao, X. P. A. Ambipolar Surface Conduction in Ternary Topological Insulator $\mathrm{Bi}_{2}\left(\mathrm{Te}_{1-\mathrm{x}} \mathrm{Se}_{\mathrm{x}}\right)_{3}$ Nanoribbons. ACS Nano 7, 2126-2131 (2013).

16. Xiu, F. et al. Manipulating Surface States in Topological Insulator Nanoribbons. Nat. Nanotechnol. 6, 216-221 (2011).

17. Ren, Z., Taskin, A. A., Sasaki, S., Segawa, K. \& Ando, Y. Large Bulk Resistivity and Surface Quantum Oscillations in the Topological Insulator $\mathrm{Bi}_{2} \mathrm{Te}_{2} \mathrm{Se}$. Phys. Rev. B 82, 241306(R) (2010).

18. Analytis, J. G. et al. Bulk Fermi Surface Coexistence with Dirac Surface State in $\mathrm{Bi}_{2} \mathrm{Se}_{3}$ : A Comparison of Photoemission and Shubnikov-de Haas Measurements. Phys. Rev. B 81, 205407 (2010).

19. Tang, H., Liang, D., R, L. J., Qiu \& Gao, P. A. X. Two-Dimensional TransportInduced Linear Magneto-Resistance in Topological Insulator $\mathrm{Bi}_{2} \mathrm{Se}_{3}$ Nanoribbons. ACS Nano 5, 7510-7516 (2011).

20. Cao, H. et al. Quantized Hall Effect and Shubnikov-de Haas Oscillations in Highly Doped $\mathrm{Bi}_{2} \mathrm{Se}_{3}$ : Evidence for Layered Transport of Bulk Carriers. Phys. Rev. Lett. 108, 216803 (2012)

21. Peng, H. et al. Aharonov-Bohm Interference in Topological Insulator Nanoribbons. Nat. Mater. 9, 225-229 (2010).

22. Li, Z. G. et al. Two-Dimensional Universal Conductance Fluctuations and the Electron-Phonon Interaction of Surface States in $\mathrm{Bi}_{2} \mathrm{Te}_{2} \mathrm{Se}$ Microflakes. Sci. Rep. 2, 595 (2012).

23. Fang, L. et al. Catalyst-Free Growth of Millimeter-Long Topological Insulator $\mathrm{Bi}_{2} \mathrm{Se}_{3}$ Nanoribbons and the Observation of the $\pi$-Berry Phase. Nano Lett. 12, 6164-6169 (2012).

24. Yan, Y., Wang, L.-X., Yu, D.-P. \& Liao, Z.-M. Large Magnetoresistance in High Mobility Topological Insulator $\mathrm{Bi}_{2} \mathrm{Se}_{3}$. Appl. Phys. Lett. 103, 033106 (2013).

25. Yan, Y. et al. Synthesis and Quantum Transport Properties of $\mathrm{Bi}_{2} \mathrm{Se}_{3}$ Topological Insulator Nanostructures. Sci. Rep. 3, 1264 (2013).

26. Xiong, J. et al. High-Field Shubnikov-de Haas Oscillations in the Topological Insulator $\mathrm{Bi}_{2} \mathrm{Te}_{2} \mathrm{Se}$. Phys. Rev. B 86, 045314 (2012).

27. Qu, D. X., Hor, Y. S., Xiong, J., Cava, R. J. \& Ong, N. P. Quantum Oscillations and Hall Anomaly of Surface States in the Topological Insulator $\mathrm{Bi}_{2} \mathrm{Te}_{3}$. Science 329, 821-824 (2010).

28. Petrushevsky, M. et al. Probing the Surface States in Bi2Se3 Using the Shubnikovde Haas Effect. Phys. Rev. B 86, 045131 (2012).

29. Ren, Z., Taskin, A. A., Sasaki, S., Segawa, K. \& Ando, Y. Large Bulk Resistivity and Surface Quantum Oscillations in the Topological Insulator $\mathrm{Bi}_{2} \mathrm{Te}_{2} \mathrm{Se}$. Phys. Rev. B 82, 241306(R) (2010).

30. Kong, D. S. et al. Rapid Surface Oxidation as a Source of Surface Degradation Factor for $\mathrm{Bi}_{2} \mathrm{Se}_{3}$. ACS Nano 5, 4698-4703 (2011).

\section{Acknowledgments}

This work was supported by MOST (Nos. 2013CB934600, 2013CB932602), NSFC (Nos. 11274014, 11234001), and the Program for New Century Excellent Talents in University of China (No. NCET-12-0002). XK and GVT acknowledge the European Research Council, ERC grant $\mathrm{N}^{\circ} 246791$ - COUNTATOMS

\section{Author contributions}

Z.M.L. conceived and designed the study. Y.Y. performed the sample growth, characterization, device fabrication, and transport measurements. X.K. and G.V.T. contributed to the HAADF-STEM measurements. X.S.W. and D.P.Y. gave scientific advice. L.X.W. and Z.M.L. wrote the manuscript. All authors contributed to discussion and reviewed the manuscript. 


\section{Additional information}

Competing financial interests: The authors declare no competing financial interests.

How to cite this article: Yan, Y. et al. High-Mobility $\mathrm{Bi}_{2} \mathrm{Se}_{3}$ Nanoplates Manifesting Quantum Oscillations of Surface States in the Sidewalls. Sci. Rep. 4, 3817; DOI:10.1038/ srep03817 (2014) (c) (i) (2) This work is licensed under a Creative Commons AttributionBY Na SA NonCommercial-ShareAlike 3.0 Unported license. To view a copy of this license, visit http://creativecommons.org/licenses/by-nc-sa/3.0 